\title{
Multi-frequency study of supernova remnants in the Large Magellanic Cloud
}

\section{The case of LMC SNR J0530-7007}

\author{
A. Y. De Horta ${ }^{1}$, M. D. Filipović ${ }^{1}$, L. M. Bozzetto ${ }^{1}$, P. Maggi ${ }^{2}$, F. Haberl ${ }^{2}$, E. J. Crawford ${ }^{1}$, M. Sasaki ${ }^{3}$, D. Urošević ${ }^{4,5}$ \\ W. Pietsch ${ }^{2}$, R. Gruendl ${ }^{6}$, J. Dickel ${ }^{7}$, N. F. H. Tothill ${ }^{1}$, Y.-H. Chu ${ }^{6}$, J. L. Payne ${ }^{1}$, and J. D. Collier ${ }^{1}$ \\ 1 School of Computing and Mathematics, University of Western Sydney Locked Bag 1797, Penrith South DC, NSW 1797, Australia \\ e-mail: a.dehorta@uws.edu.au \\ 2 Max-Planck-Institut für extraterrestrische Physik, Giessenbachstraße, 85748 Garching, Germany \\ 3 Institut für Astronomie und Astrophysik Tübingen, Sand 1, 72076 Tübingen, Germany \\ ${ }^{4}$ Department of Astronomy, Faculty of Mathematics, University of Belgrade, Studentski trg 16, 11000 Belgrade, Serbia \\ 5 Isaac Newton Institute of Chile, Yugoslavia Branch, Belgrade, Serbia \\ ${ }^{6}$ Department of Astronomy, University of Illinois, 1002 West Green Street, Urbana, IL 61801, USA \\ 7 Physics and Astronomy Department, University of New Mexico, MSC 07-4220, Albuquerque, NM 87131, USA
}

Received 21 December 2011 / Accepted 8 February 2012

\section{ABSTRACT}

\begin{abstract}
Context. The supernova remnants (SNRs) known in the Large Magellanic Cloud (LMC) show a variety of morphological structures in the different wavelength bands. This variety is the product of the conditions in the surrounding medium with which the remnant interacts and the inherent circumstances of the supernova event itself.

Aims. This paper performs a multi-frequency study of the LMC SNR J0530-7007 by combining Australia Telescope Compact Array (ATCA), Molonglo Observatory Synthesis Telescope (MOST), Röntgensatellit (ROSAT) and Magellanic Clouds Emission Line Survey (MCELS) observations.

Methods. We analysed radio-continuum, X-ray and optical data and present a multi-wavelength morphological study of LMC SNR J0530-7007.

Results. We find that this object has a shell-type morphology with a size of $215^{\prime \prime} \times 180^{\prime \prime}(52 \mathrm{pc} \times 44 \mathrm{pc})$; a radio spectral index $(\alpha=-0.85 \pm 0.13)$; with $[\mathrm{S}$ II] $/ \mathrm{H} \alpha>0.4$ in the optical; and the presence of non-thermal radio and X-ray emission.

Conclusions. We confirmed this object as a bona-fide shell-type SNR which is probably a result of a Type Ia supernova.
\end{abstract}

Key words. ISM: supernova remnants - Magellanic Clouds - radio continuum: ISM - ISM: individual objects: SNR J0530-7007

\section{Introduction}

Lying towards the south ecliptic pole, the Large Magellanic Cloud (LMC), is in one of the coldest parts of the radio sky, uncontaminated by Galactic foreground emission (Haynes et al. 1991). The LMC's position and its known distance of $50 \mathrm{kpc}$ (di Benedetto 2008) makes the LMC arguably the best galaxy in which to study supernova remnants (SNRs) in our Local Group of galaxies.

In the radio-continuum, SNR emission is predominantly non-thermal, giving rise to a typical radio spectral index of $\alpha \sim-0.5\left(S \propto v^{\alpha}\right)$. However, the environment in which the SNR evolves i.e. the interstellar medium (ISM) with its ambient magnetic field, will not only affect the radio spectral index observed but also the SNR's morphology, structure and behaviour (Filipović et al. 1998).

In a $\mathrm{H} \alpha$ survey of the LMC, Davies et al. (1976) reported "diffuse filaments" with a size of $10^{\prime} \times 9^{\prime}$ at $\mathrm{RA}(\mathrm{J} 2000)=5^{\mathrm{h}} 30^{\mathrm{m}} 30 \mathrm{~s} 35$ and $\operatorname{Dec}(\mathrm{J} 2000)=-70^{\circ} 07^{\prime} 51^{\prime \prime} .5$ and named it DEM L218. A radio source designated 0531-701 was identified by Turtle \& Mills (1984) at RA(J2000) $=5^{\mathrm{h}} 30^{\mathrm{m}} 38^{\mathrm{s}} .19$ and $\operatorname{Dec}(\mathrm{J} 2000)=-70^{\circ} 07^{\prime} 33^{\prime \prime}$ and classified as an SNR candidate in a survey with the Molonglo Observatory Synthesis Telescope (MOST). Filipović et al. (1998) detected this source in the Parkes radio surveys of the Magellanic Clouds (MCs; $\lambda=6 \mathrm{~cm}$ and $3 \mathrm{~cm}$ ) but due to a rather flat spectrum of $\alpha=-0.17 \pm 0.24$ and the survey's low resolution (Parkes Beam Sizes: 4.9 at $\lambda=6 \mathrm{~cm} ; 2.7$ at $\lambda=3 \mathrm{~cm}$ ), they were unable to classify it as an SNR at that time. Haberl \& Pietsch (1999, hereafter HP99) detected a nearby Röntgensatellit (ROSAT) X-ray source ([HP99] 1081) at a position of RA $(J 2000)=05^{\mathrm{h}} 30^{\mathrm{m}} 51.8^{\mathrm{s}}$ and $\operatorname{Dec}(\mathrm{J} 2000)=-70^{\circ} 06^{\prime} 44^{\prime \prime}$; however, this object is close to the LMC bar where confusion is significant. The object was rediscovered using the Magellanic Clouds Emission Line Survey (MCELS) (Smith et al. 2004). This study inferred that the object is likely a large, old radiative shell-type SNR with enhanced [S II] ([S II]/H $\alpha>0.4)$. Blair et al. (2006) also observed the object using the Far Ultraviolet Spectroscopic Explorer (FUSE) satellite, at a position of $\mathrm{RA}(\mathrm{J} 2000)=05^{\mathrm{h}} 30^{\mathrm{m}} 37^{\mathrm{s}}$, $\operatorname{Dec}(\mathrm{J} 2000)=-70^{\circ} 08^{\prime} 40^{\prime \prime}$ with a beamsize of $145^{\prime \prime}$. Weak, yet moderately broad lines of $\mathrm{O}$ vi were detected, in addition to possible, but uncertain C III lines. Desai et al. (2010) reported that there are no molecular clouds detected towards this object and that there are no young stellar objects in its vicinity.

In this paper, we report new Australia Telescope Compact Array (ATCA) radio-continuum observations at at $\lambda=3 \mathrm{~cm}$ and $6 \mathrm{~cm}$. These new radio-continuum observations in conjunction with previous radio-continuum $(\lambda=20 \mathrm{~cm}$ and $13 \mathrm{~cm}$ 
Table 1. Integrated flux densities of SNR J0530-7007.

\begin{tabular}{ccccc}
\hline \hline $\begin{array}{l}v \\
(\mathrm{MHz})\end{array}$ & $\begin{array}{c}\lambda \\
(\mathrm{cm})\end{array}$ & $\begin{array}{c}\text { Beam size } \\
\left({ }^{\prime \prime}\right)\end{array}$ & $\begin{array}{c}\mathrm{rms} \\
(\mathrm{mJy})\end{array}$ & $\begin{array}{c}\mathrm{S}_{\text {Total }} \\
(\mathrm{mJy})\end{array}$ \\
\hline 843 & 36 & $43.0 \times 43.0$ & 0.5 & $107^{a}$ \\
843 & 36 & $43.0 \times 43.0$ & 0.5 & 80 \\
1400 & 20 & $40.0 \times 40.0$ & 0.5 & 62 \\
2400 & 13 & $54.1 \times 48.9$ & 0.4 & 52 \\
5500 & 6 & $33.8 \times 33.8$ & 0.05 & 23 \\
9000 & 3 & $22.5 \times 22.5$ & 0.05 & 10 \\
\hline
\end{tabular}

Notes. ${ }^{(a)}$ Integrated flux density from Turtle \& Mills (1984).

(ATCA), $\lambda=36 \mathrm{~cm}($ MOST)), X-ray (ROSAT) and optical (MCELS) observations are used to confirm that the object in the LMC centered at $\mathrm{RA}(\mathrm{J} 2000)=5^{\mathrm{h}} 30^{\mathrm{m}} 40.4^{\mathrm{s}}$ and $\operatorname{Dec}(\mathrm{J} 2000)=-70^{\circ} 07^{\prime} 27.4^{\prime \prime}$ is a bona-fide SNR that, hereafter, we will call SNR J0530-7007. The observations, data reduction and imaging techniques are described in Sect. 2. Astrophysical interpretation of the newly obtained moderate-resolution total intensity images, in combination with existing ROSAT and MCELS images are discussed in Sect. 3.

\section{Observations and data reduction}

\subsection{Radio-continuum}

Radio-continuum observations at the five frequencies shown in Table 1 have been used to study and measure the flux densities of SNR J0530-7007. For the $36 \mathrm{~cm}$ (MOST) flux density measurement given in Table 1, we used the unpublished image as described by Mills et al. (1984). The $20 \mathrm{~cm}$ (ATCA) image used is from Hughes et al. (2007).

Australia Telescope Compact Array (ATCA) observations from 1992 April 29 and 30 (project C195) of SNR J0530-7007 were also used. These observations were made at wavelengths $\lambda=13 \mathrm{~cm}$ and $20 \mathrm{~cm}(v=2400 \mathrm{MHz}$ and $1400 \mathrm{MHz})$ using the 375 array configuration.

Recently, on 2011 November 15, we observed SNR J0530-7007 (project C634), with the ATCA in the EW352 configuration, at $\lambda=3 / 6 \mathrm{~cm}(9000$ and $5500 \mathrm{MHz})$ and a bandwidth of $2 \mathrm{GHz}$ (ATCA project C634). In addition to this, SNR J0530-7007 was observed using the ATCA on 1995 November 12 with the 6A array (ATCA Project C461; $\lambda=3 / 6 \mathrm{~cm}(v=8640 \mathrm{MHz}$ and $4800 \mathrm{MHz}))$. Both observations were carried out in snap-shot mode, totalling about $1 \mathrm{hr}$ (each) of integration over a $12 \mathrm{~h}$ period. These two sets of observations were combined with mosaic observations from project C918 (Dickel et al. 2005).

For all these radio-continuum observations, baselines formed with ATCA antenna 6 were excluded, as the other five antennas were arranged in a compact configuration. Source PKS B1934-638 was used for primary (flux) calibration and source PKS B0530-727 was used for secondary (phase) calibration. More information on the observing procedure and other sources observed under the project C634 can be found in Bojičić et al. (2007); Crawford et al. (2008a,b); Čajko et al. (2009); Crawford et al. (2010); Bozzetto et al. (2010, 2011, 2012). Parkes radio-continuum data from Filipović et al. (1995, 1996) were initially combined with the ATCA data wherever the shortest ATCA baselines were less than the Parkes diameter of $64 \mathrm{~m}$, and the observational frequencies corresponded, in order to provide zero-spacing. However, as the rms noise of the Parkes

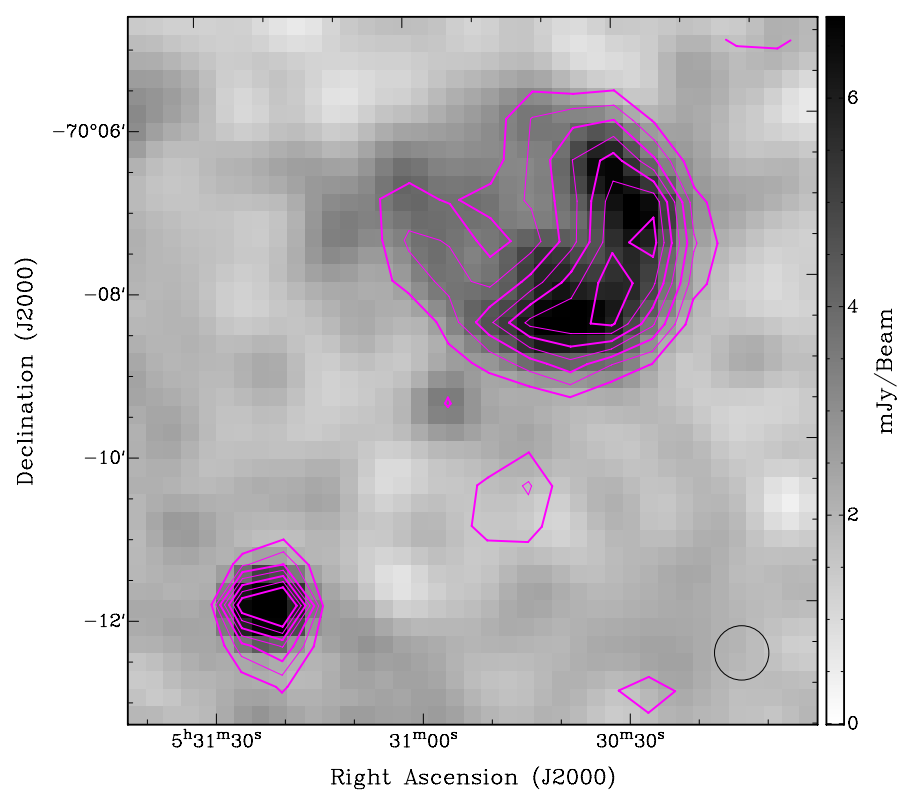

Fig. 1. Combined ATCA observations of SNR J0530-7007 at $20 \mathrm{~cm}$ (1400 MHz) overlaid with MOST $36 \mathrm{~cm}(843 \mathrm{MHz})$ contours. Contours are from 2 to $8 \mathrm{mJy} / \mathrm{beam}$ in steps of $1 \mathrm{mJy} / \mathrm{beam}$. The black circle in the lower right corner represents the synthesised beamwidth (at $20 \mathrm{~cm}$ ) of $40^{\prime \prime}$.

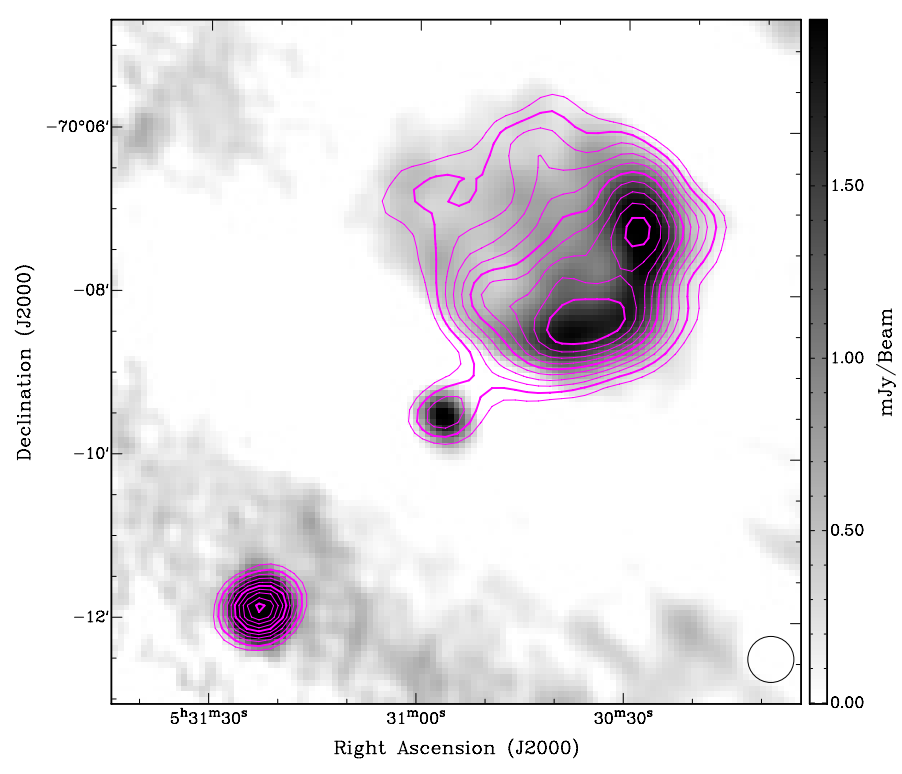

Fig. 2. Combined ATCA observations of SNR J0530-7007 at $6 \mathrm{~cm}$ (5500 MHz) overlaid with $13 \mathrm{~cm}(2400 \mathrm{MHz})$ contours. Contours are from 1.5 to $6 \mathrm{mJy} /$ beam in steps of $0.5 \mathrm{mJy} / \mathrm{beam}$. The black circle in the lower right corner represents the synthesised beamwidth (at $6 \mathrm{~cm}$ ) of $33.8^{\prime \prime}$.

observations are significantly higher, especially at $3 / 6 \mathrm{~cm}$, it was decided not to use these observations in our final analysis.

The total-intensity images in Figs. 1 and 2 were formed using the standard MIRIAD (Sault et al. 1995) tasks employing multifrequency synthesis using a natural weighting scheme with a correction for the primary beam response applied. A similar procedure was used for both $U$ and $Q$ Stokes parameter maps. However, due to the low dynamic-range (signal to noise ratio between the source flux and $3 \sigma$ noise level) self-calibration could not be applied. 


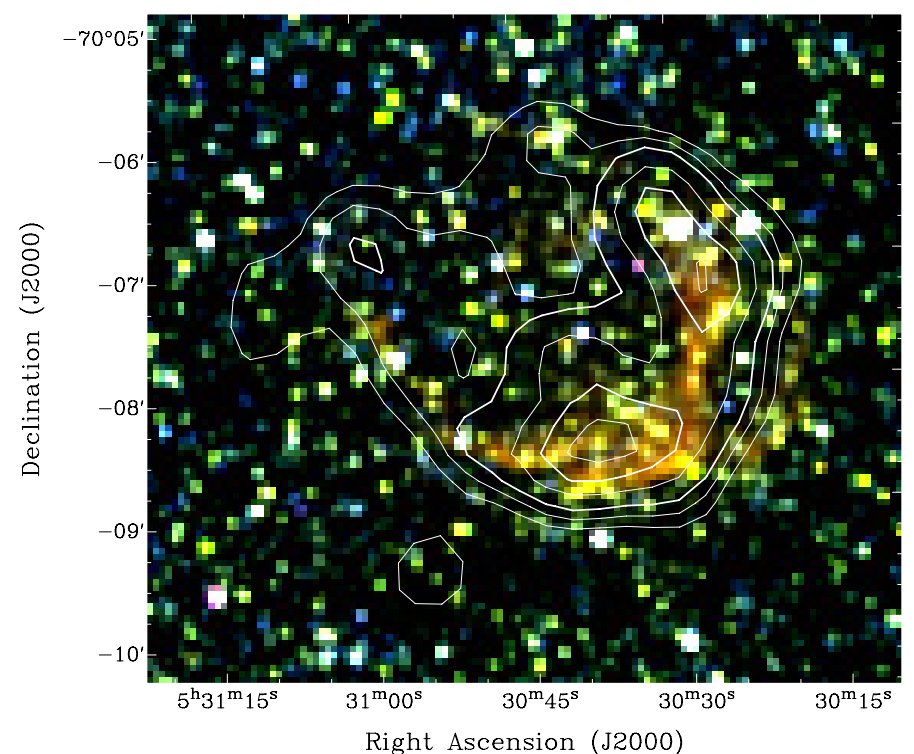

Fig. 3. MCELS composite optical image ( $\mathrm{RGB}=\mathrm{H} \alpha$, [SII],[OIII]) of SNR J0530-7007 overlaid with $20 \mathrm{~cm}$ radio contours. Contours are 3, 3.5, 4, 5, 6 and $7 \mathrm{mJy} / \mathrm{beam}$.

\subsection{Optical}

The MCELS observations (Smith et al. 2006) were carried out with the $0.6 \mathrm{~m}$ University of Michigan/Cerro Tololo Inter-American Observatory (CTIO) Curtis Schmidt telescope, equipped with a SITe $2048 \times 2048 \mathrm{CCD}$, giving a field of $1.35^{\circ} \times 1.35^{\circ}$ at a pixel scale of $2.4^{\prime \prime} \times 2.4^{\prime \prime}$. It mapped both the LMC and SMC in narrow bands covering $\mathrm{H} \alpha$, [O III] $(\lambda=$ $5007 \AA)$, and [S II] $(\lambda=6716,6731 \AA)$. Also observed were matched red and green continuum bands, used primarily to subtract the stars from the images to reveal the full extent of the faint diffuse emission. All the data has been flux-calibrated and assembled into mosaic images; a small section of the mosaic is shown in Fig. 3.

The high-resolution $\mathrm{H} \alpha$ image in Fig. 3 was obtained with the MOSAIC II camera on the Blanco 4-m telescope at the CTIO. It confirms a distinctive optical nebulosity associated with the SNR candidate. Here, for the first time, we present optical images of this object in combination with our new ATCA radio-continuum and ROSAT X-ray data.

\subsection{X-rays}

SNR J0530-7007 lies in the field of view of several pointed observations of ROSAT's Position Sensitive Proportional Counter (PSPC). The HP99 catalogue includes a very weak detection ([HP99] 1081, Fig. 4) within the extent of the radio emission from SNR J0530-7007. However, the X-ray properties of this source are unclear: The hardness ratios are poorly defined $(\mathrm{HR} 1=1.00 \pm 1.23)$ or undefined $(\mathrm{HR} 2)$ and therefore give no meaningful information on the spectrum. The HP99 catalogue was derived from individual PSPC observations, without combining the exposures of overlapping fields. To investigate [HP99] 1081 and its possible association with SNR J0530-7007 in more detail, we selected 9 observations of the LMC which covered the SNR within $24^{\prime}$ of the optical axis (to avoid the degraded point spread function at larger off-axis angles). In Table 2 we give the ROSAT sequence number, target name, exposure time and central coordinates of the selected pointings, as well as

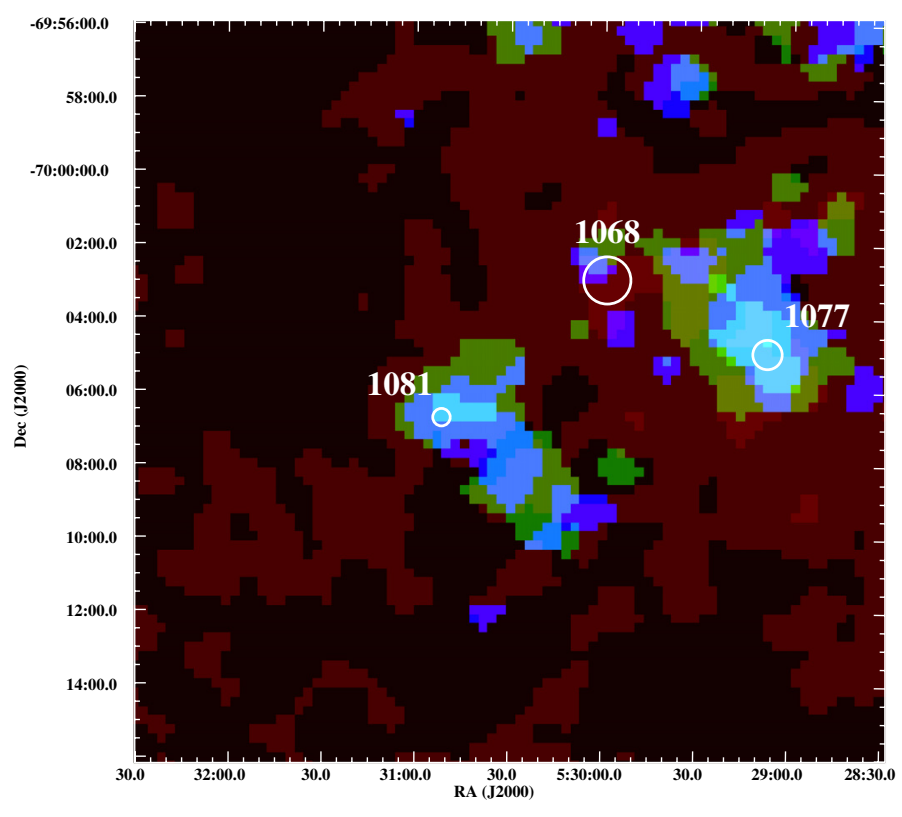

Fig. 4. The ROSAT PSPC RGB colour image of the area around SNR J0530-7007. The energy bands are: red (0.1-0.4 keV), green $(0.5-0.9 \mathrm{keV})$ and blue $(0.9-2.0 \mathrm{keV})$. The image has a pixel size of $15^{\prime \prime}$ and is smoothed with a $\sigma$ of 1.5 pixel. The annotations denote sources from HP99.

the off-axis angle of SNR J0530-7007 in each of them. Images were produced at different energy bands (broad: $0.1-2.4 \mathrm{keV}$, soft: $0.1-0.4 \mathrm{keV}$, hard: $0.5-2.0 \mathrm{keV}$, hard1: $0.5-0.9 \mathrm{keV}$ and hard2: $0.9-2.0 \mathrm{keV}$ ) from the merged data. A colour image of the area around the SNR with net exposure (vignetting corrected) of $\sim 48 \mathrm{ks}$ is shown in Figs. 4 and 5 with red, green and blue representing the X-ray intensities in the soft, hard 1 and hard 2 bands. The resolution of the ROSAT PSPC varies with energy but the point spread function is always less than $1^{\prime}$.

The HP99 catalogue contains two other sources detected in the neighbourhood of source 1081 (see Fig. 4). [HP99] 1068 is a weak source with an existence likelihood of 11.8, just above the threshold used for the catalogue. No useful information about extent or hardness ratios can be derived. Source [HP99] 1077 is a clear detection (existence likelihood 22.8) with indication for an extent of 31"(likelihood for the extent of 13.3). Therefore, [HP99] 1077 looks like an extended source itself, but no significant radio nor optical emission in the MCELS images is seen at its position. Future X-ray observations are required to investigate the whole region around the three ROSAT sources in more detail.

\section{Results and discussion}

The remnant has a typical horse-shoe morphology (Figs. 1, 2 and 3), centered at $\mathrm{RA}(\mathrm{J} 2000)=5^{\mathrm{h}} 30^{\mathrm{m}} 40.4^{\mathrm{s}}$ and $\operatorname{Dec}(\mathrm{J} 2000)=$ $-70^{\circ} 07^{\prime} 27.4^{\prime \prime}$. The measured position differs from that of the FUSE observations (Blair et al. 2006), since the FUSE observation was aimed only at the Southern side of the shell and not at the centre of the SNR.

The size of SNR J0530-7007 at $\lambda=20 \mathrm{~cm}$ is $215^{\prime \prime} \pm 4^{\prime \prime}$ $\times 180^{\prime \prime} \pm 4^{\prime \prime}(52 \pm 1 \mathrm{pc} \times 44 \pm 1 \mathrm{pc})$. The size was measured by taking line profiles along the major (NE-SW) and minor (SE$\mathrm{NW}$ ) axis $\left(\mathrm{PA}=45^{\circ}\right)$ of the remnant using the KARMA ${ }^{1}$ (Gooch 2006) tool KPVSLICE and detemining the distance between the

${ }^{1}$ http://www.atnf.csiro.au/computing/software/karma/ 
Table 2. ROSAT observations summary of SNR J0530-7007 (sorted by RA).

\begin{tabular}{llcccc}
\hline \hline $\begin{array}{l}\text { ROSAT } \\
\text { sequence }\end{array}$ & Target name & $\begin{array}{c}\text { Obs. time } \\
(\mathrm{s})\end{array}$ & RA & Dec & $\begin{array}{c}\text { Off-axis angle }^{a} \\
(\mathrm{~J} 2000)\end{array}$ \\
\hline $180287 \mathrm{p}$ & NOVA LMC 1995 & 2531 & $05^{\mathrm{h}} 26^{\mathrm{m}} 50.04^{\mathrm{s}}$ & $-70^{\circ} 01^{\prime} 11.5^{\prime \prime}$ & 21.3 \\
$400298 \mathrm{p}$ & RX J0527.8-6954 & 1058 & $05^{\mathrm{h}} 27^{\mathrm{m}} 48.00^{\mathrm{s}}$ & $-69^{\circ} 54^{\prime} 00.0^{\prime \prime}$ & 20.2 \\
$400298 \mathrm{p}-1$ & RX J0527.8-6954 & 7502 & $05^{\mathrm{h}} 27^{\mathrm{m}} 48.00^{\mathrm{s}}$ & $-69^{\circ} 54^{\prime} 00.0^{\prime \prime}$ & 20.2 \\
$400298 \mathrm{p}-2$ & RX J0527.8-6954 & 7802 & $05^{\mathrm{h}} 27^{\mathrm{m}} 48.00^{\mathrm{s}}$ & $-69^{\circ} 54^{\prime} 00.0^{\prime \prime}$ & 20.2 \\
$400148 \mathrm{p}$ & RX J0527.8-6954 & 6064 & $05^{\mathrm{h}} 27^{\mathrm{m}} 48.00^{\mathrm{s}}$ & $-69^{\circ} 54^{\prime} 00.0^{\prime \prime}$ & 20.2 \\
$180255 \mathrm{p}$ & RX J0527.8-6954 & 9763 & $05^{\mathrm{h}} 27^{\mathrm{m}} 50.04^{\mathrm{s}}$ & $-69^{\circ} 54^{\prime} 00.0^{\prime \prime}$ & 20.0 \\
$300172 \mathrm{p}$ & NOVA LMC 1988 a & 6272 & $05^{\mathrm{h}} 32^{\mathrm{m}} 28.08^{\mathrm{s}}$ & $-70^{\circ} 21^{\prime} 36.0^{\prime \prime}$ & 17.0 \\
$300172 \mathrm{p}-1$ & NOVA LMC 1988 a & 2993 & $05^{\mathrm{h}} 32^{\mathrm{m}} 28.08^{\mathrm{s}}$ & $-70^{\circ} 21^{\prime} 36.0^{\prime \prime}$ & 17.0 \\
$300172 \mathrm{p}-2$ & NOVA LMC 1988 a & 3880 & $05^{\mathrm{h}} 32^{\mathrm{m}} 28.08^{\mathrm{s}}$ & $-70^{\circ} 21^{\prime} 36.0^{\prime \prime}$ & 17.0 \\
\hline
\end{tabular}

Notes. ${ }^{(a)}$ Mean angular distance of [HP99] 1081 to the optical axis of the telescope.

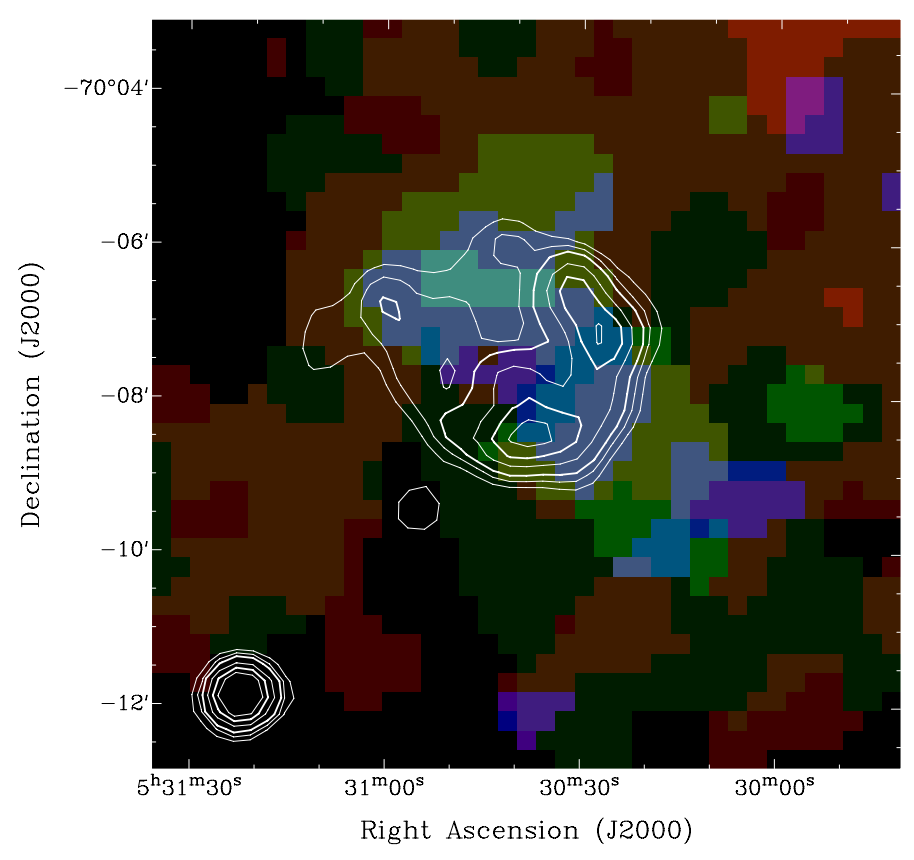

Fig. 5. The ROSAT PSPC RGB colour image of SNR J0530-7007 overlaid with the contours of $20 \mathrm{~cm}$ intensity. Contours are 3, 3.5, 4, 5, 6 and $7 \mathrm{mJy} / \mathrm{beam}$.

point when the line profile first rises above $3 \sigma(1.50 \mathrm{mJy})$ and the point when it finally falls below $3 \sigma$. The thickness of the shell is estimated to be $<30^{\prime \prime}(7 \mathrm{pc}$ ) at $6 \mathrm{~cm}$, about $30 \%$ of the SNR's radius (ie. a filling factor of 0.64 ).

The merged ROSAT PSPC images reveal an elongated structure of X-ray emission at the location of the SNR with a brighter spot right at the position of [HP99] 1081. The presence of extended X-ray emission is coincident with the radio emission of the SNR (Fig. 5). In X-rays, the brightest part is in the northeast while the MCELS optical emission closely follows radiocontinuum appearance (Fig. 3).

We note that the significant difference in the flux density measurement at $36 \mathrm{~cm}$ (107 mJy in Turtle \& Mills (1984) vs $80 \mathrm{mJy}$ in this work) may introduce a very large uncertainty in the spectral index measurement $\left(S \propto v^{\alpha}\right)$. A possible explanation for this discrepancy is that we applied different fitting model then Turtle \& Mills (1984). Using all values of integrated flux density estimates (except for $36 \mathrm{~cm}$ value from Turtle \& Mills (1984); Table 1), a spectral index $\left(S \propto v^{\alpha}\right)$ distribution is plotted in Fig. 6. The overall radio-continuum spectra (Fig. 6; black line)

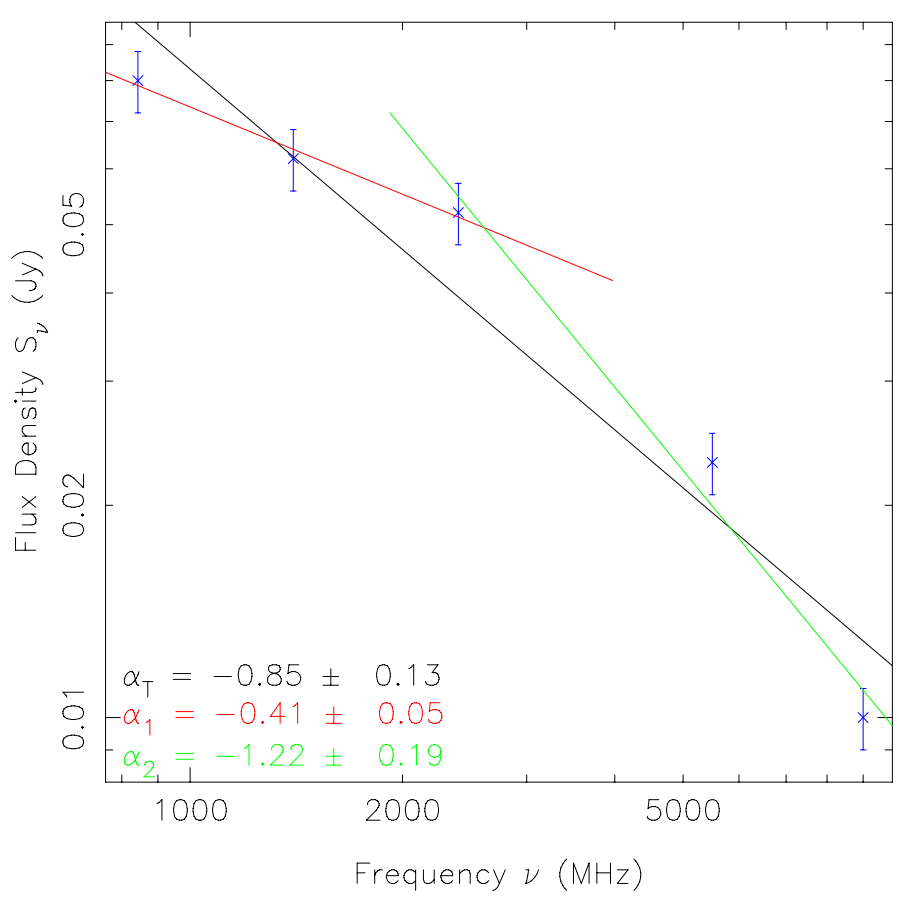

Fig. 6. Radio-continuum spectrum of SNR J0530-7007. The black line $\left(\alpha_{\mathrm{T}}\right)$ is the overall radio-continuum spectra, the red line $\left(\alpha_{1}\right)$ between $36 \mathrm{~cm}$ and $13 \mathrm{~cm}$, and the green line $\left(\alpha_{2}\right)$ between $13 \mathrm{~cm}$ and $3 \mathrm{~cm}$. Note $\log -\log$ scale.

from SNR J0530-7007 was estimated to be $\alpha_{\mathrm{T}}=-0.85 \pm 0.13$, while the typical SNR spectral index is $\alpha=-0.5 \pm 0.2$ (Filipović et al. 1998). This somewhat steeper spectral index would indicate a younger age despite its (large) size of $52 \times 44 \mathrm{pc}$, suggesting it as an older (more evolved) SNR. We also note that this may indicate that a simple model does not accurately describe the data, and that a higher order model is needed. This is not unusual, given that several other Magellanic Clouds SNR's exhibit this "curved" spectra (Crawford et al. 2008a; Bozzetto et al. 2010, 2011). Noting the breakdown of the power law fit at shorter wavelengths, we decomposed the spectral index estimate into two components, one $\left(\alpha_{1}\right)$ between 36 and $13 \mathrm{~cm}$, and the other $\left(\alpha_{2}\right)$ between 13 and $3 \mathrm{~cm}$. The first component (Fig. 6; red line), $\alpha_{1}=-0.41 \pm 0.05$ is a reasonable fit and typical for an evolved SNR, whereas the second (Fig. 6; green line), $\alpha_{2}=-1.22 \pm 0.19$, is a poor fit, and indicates that nonthermal emission can be described by different populations of electrons with different energy indices. Although the low flux at 


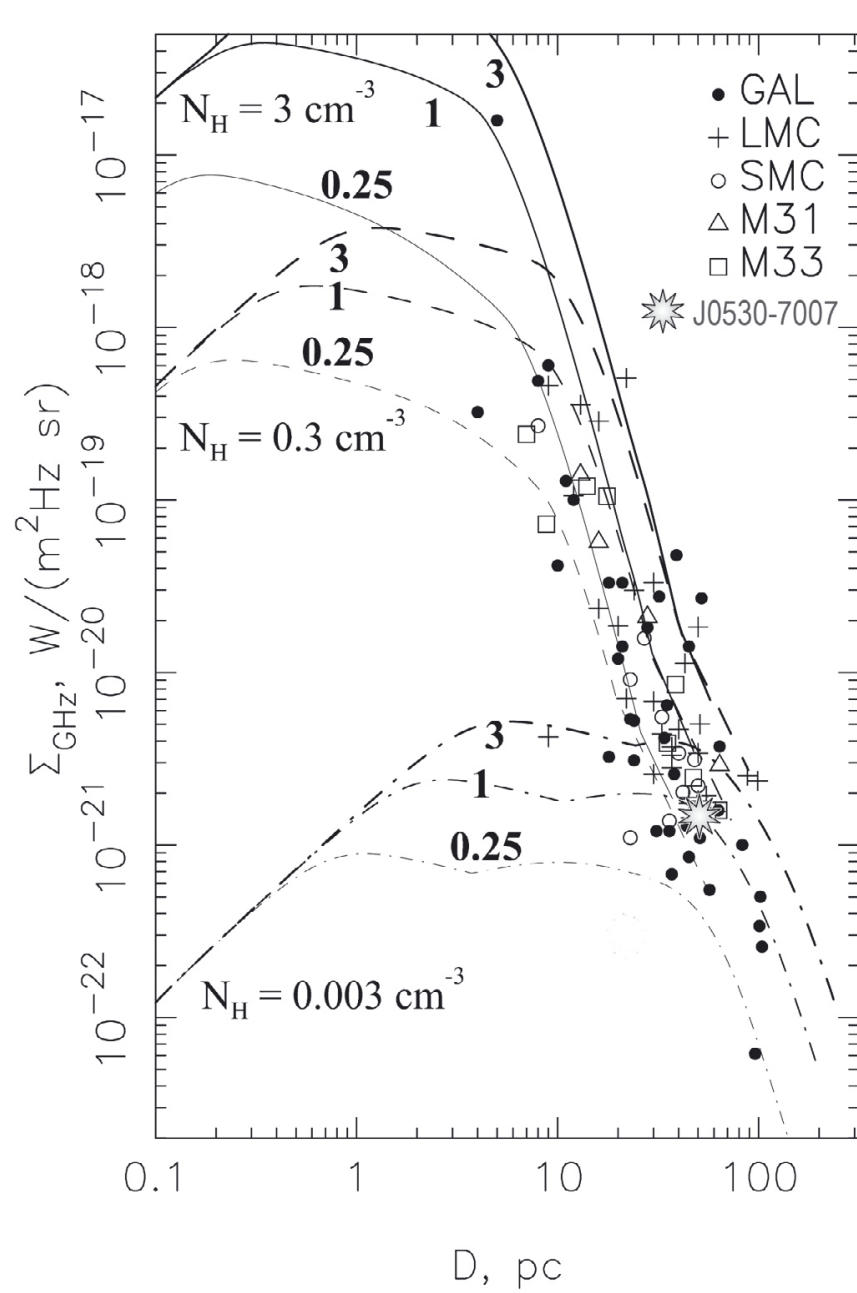

Fig. 7. $1 \mathrm{GHz}$ Surface brightness-to-diameter diagram from Berezhko \& Völk (2004), with SNR J0530-7007 added. The evolutionary tracks are for ISM densities of $N_{\mathrm{H}}=3,0.3$ and $0.003 \mathrm{~cm}^{-3}$ and explosion energies of $E_{\mathrm{SN}}=0.25,1$ and $3 \times 10^{51} \mathrm{erg}$.

$3 \mathrm{~cm}$ (and to a lesser extent at $6 \mathrm{~cm}$ ) could cause the large deviations, an underestimate of up to $\sim 50 \%$ would still lead to a "curved" spectrum.

Without reliable polarisation measurements at any radiocontinuum frequency we cannot determine the Faraday rotation and thus cannot deduce the magnetic field strength. However, we can use the new equipartion formula derived by Arbutina et al. (2012) from diffusive shock acceleration (DSA) theory (Bell 1978) to estimate a magnetic field strength: This formula is particularly relevant to magnetic field estimation in SNRs, and yields magnetic field strengths, similar to but more precise than, those given by classical equipartition (Pacholczyk 1970) and the revised equipartition (Beck \& Krause 2005) method. The average equipartition field over the whole shell of SNR J0530-7007 is $\sim 53 \mu \mathrm{G}$ (see Arbutina et al. (2012); and corresponding "calculator" ${ }^{\prime 2}$, corresponding those of middle-aged SNRs where the interstellar magnetic field is compressed and moderately amplified by the strong shocks.

Figure 7 shows a surface brightness-diameter $(\Sigma-D)$ diagram at $1 \mathrm{GHz}$ with theoretically-derived evolutionary tracks (Berezhko \& Völk 2004) superposed. SNR J0530-7007 lies at

\footnotetext{
${ }^{2}$ Calculator available at http://poincare.matf.bg.ac.rs/ $\sim$ arbo/eqp/
}

$(D, \Sigma)=\left(48 \mathrm{pc}, 1.1 \times 10^{-21} \mathrm{~W} \mathrm{~m}^{-2} \mathrm{~Hz}^{-1} \mathrm{Sr}^{-1}\right)$ on the diagram. Its position tentatively suggests that it is in the early Sedov phase of evolution - expanding into a very low density environment with the canonical initial energy of a supernova explosion $\left(10^{51} \mathrm{erg}\right)$.

High-mass stars rarely form in isolation, so core-collapse supernovae are expected to be associated with other high-mass stars. We used data from the Magellanic Cloud Photometric Survey (MCPS Zaritsky et al. 2004) to construct colourmagnitude diagrams (CMDs) and identify blue stars more massive than $\sim 8 M_{\odot}$ within a $100 \mathrm{pc}\left(396^{\prime \prime}\right)$ radius of SNR J0530-7007. The CMD in Fig. 8 (left) contains only 13 B-star candidates $(V<16, B-V<0)$. The red crosses in Fig. 8 (right) shows where the B-star candidates are with respect to SNR J0530-7007. These criteria would also find stars as late as B2-3 stars. More stringent criteria $(V<14, B-V<0)$, roughly equivalent to searching for $\mathrm{OB}$ stars in the Sanduleak (1970) catalog, would find only 1 star.

Comparison of the star formation histories (Harris \& Zaritsky 2009) in the vicinity of SNR J0530-7007 and SNR J0529-6654 (Bozzetto et al., in press) yields a significant difference: The star formation rate near SNR J0530-7007 shows an upturn around $50 \mathrm{Myr}$ ago, whereas the vicinity of SNR J0529-6654 has a strong spike in the star formation rate in the last 12-25 Myr. The lack of recent high-mass star formation around SNR J0530-7007 suggests that it is more likely to be the remnant of a Type Ia supernova.

\section{Conclusion}

We have carried out the first detailed multi-frequency study of the LMC SNR J0530-7007, showing that:

1. SNR J0530-7007 is a relatively large $\left(215^{\prime \prime} \pm 4^{\prime \prime} \times\right.$ $\left.180^{\prime \prime} \pm 4^{\prime \prime}(52 \pm 1 \mathrm{pc} \times 44 \pm 1 \mathrm{pc})\right)$ shell-type SNR;

2. It has radio spectral index $\alpha=-0.85 \pm 0.13$ between $843 \mathrm{MHz}$ and $9000 \mathrm{MHz}$, but the spectrum appears to be peaked/curved;

3. SNR J0530-7007 is in the early Sedov phase, expanding into a very low density environment;

4. The average equipartition field over the whole shell of SNR J0530-7007 is $\sim 53 \mu \mathrm{G}$;

5. There is a lack of recent local high-mass star formation, suggesting that SNR J0530-7007 is the remnant of a Type Ia supernova.

With strong optical [S II] emission ([S II]/H $\alpha>0.4)$, the presence of non-thermal radio and X-ray emission, this object satisfies all three criteria for classifying it as an SNR.

Acknowledgements. We used the KARMA and MIRIAD software package developed by the ATNF. The ATCA is part of the Australia Telescope which is funded by the Commonwealth of Australia for operation as a National Facility managed by CSIRO. The Magellanic Clouds Emission Line Survey (MCELS) data are provided by R. C. Smith, P. F. Winkler, and S. D. Points. The MCELS project has been supported in part by NSF grants AST-9540747 and AST-0307613, and through the generous support of the Dean B. McLaughlin Fund at the University of Michigan, a bequest from the family of Dr. Dean B. McLaughlin in memory of his lasting impact on Astronomy. The National Optical Astronomy Observatory is operated by the Association of Universities for Research in Astronomy Inc. (AURA), under a cooperative agreement with the National Science Foundation. This research is supported by the Ministry of Education and Science of the Republic of Serbia through project No. 176005. P. Maggi acknowledges support 

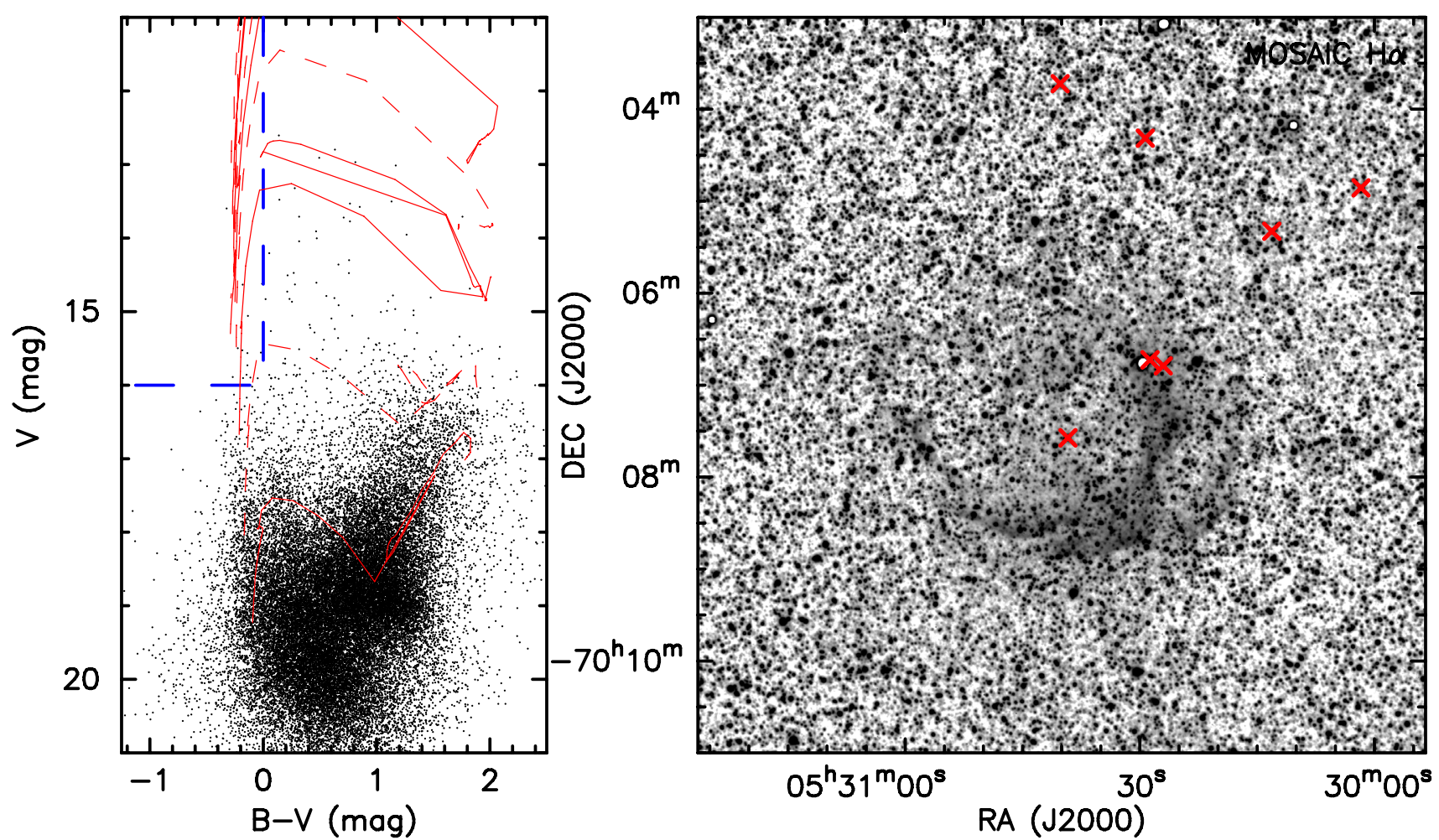

Fig. 8. The left panel shows a $B-V, V$ colour-magnitude diagram from the MCPS (Zaritsky et al. 2004). Stellar evolutionary tracks from Lejeune \& Schaerer (2001) are shown as dashed red lines (bottom to top: 5, 15, 25 and $60 M_{\odot}$ ) and solid red lines (bottom to top: 3, 9, 20 and $40 M_{\odot}$ ). Heavy dashed blue lines denote the selection criteria for B-star candidates, which lie in the top left corner of the diagram. The right panel shows the MCELS-2 H $\alpha$ image of the area around SNR J0530-7007. B-star candidates are denoted by overlaid red crosses.

from the Bundesministerium für Wirtschaft und Technologie/Deutsches Zentrum für Luft- und Raumfahrt ( BMWI/DLR) grant FKZ 50 OR 1201.

\section{References}

Arbutina, B., Urosević, D., Andjelić, M. M., Pavlović, M. Z., \& Vukotić, B. 2012, ApJ, 746, 79

Beck, R., \& Krause, M. 2005, Astron. Nachr., 326, 414

Bell, A. R. 1978, MNRAS, 182, 443

Berezhko, E. G., \& Völk, H. J. 2004, A\&A, 427, 525

Blair, W. P., Ghavamian, P., Sankrit, R., \& Danforth, C. W. 2006, ApJS, 165, 480 Bojičić, I. S., Filipović, M. D., Parker, Q. A., et al. 2007, MNRAS, 378, 1237

Bozzetto, L. M., Filipović, M. D., Crawford, E. J., et al. 2010, Serbian Astron. J., 181, 43

Bozzetto, L. M., Filipović, M. D., Crawford, E. J., et al. 2011, Rev. Mex. Astron. Astrofis., submitted [arXiv: 1109.3945]

Bozzetto, L. M., Filipović, M. D., Crawford, E. J., et al. 2012, MNRAS, 420, 2588

Čajko, K. O., Crawford, E. J., \& Filipović, M. D. 2009, Serbian Astron. J., 179, 55

Crawford, E. J., Filipović, M. D., De Horta, A. Y., Stootman, F. H., \& Payne, J. L. 2008a, Serbian Astron. J., 177, 61

Crawford, E. J., Filipović, M. D., \& Payne, J. L. 2008b, Serbian Astron. J., 176, 59

Crawford, E. J., Filipović, M. D., Haberl, F., et al. 2010, A\&A, 518, A35

Davies, R. D., Elliott, K. H., \& Meaburn, J. 1976, MmRAS, 81, 89
Desai, K. M., Chu, Y.-H., Gruendl, R. A., et al. 2010, AJ, 140, 584 di Benedetto, G. P. 2008, MNRAS, 390, 1762

Dickel, J. R., McIntyre, V. J., Gruendl, R. A., \& Milne, D. K. 2005, AJ, 129, 790 Filipović, M. D., Haynes, R. F., White, G. L., et al. 1995, A\&AS, 111, 311 Filipović, M. D., White, G. L., Haynes, R. F., et al. 1996, A\&AS, 120, 77

Filipović, M. D., Haynes, R. F., White, G. L., \& Jones, P. A. 1998, A\&AS, 130, 421

Gooch, R. 2006, Karma Users Manual (Australia Telescope National Facility) Haberl, F., \& Pietsch, W. 1999, A\&AS, 139, 277 (HP99)

Harris, J., \& Zaritsky, D. 2009, AJ, 138, 1243

Haynes, R. F., Klein, U., Wayte, S. R., et al. 1991, A\&A, 252, 475

Hughes, A., Staveley-Smith, L., Kim, S., Wolleben, M., \& Filipović, M. 2007, MNRAS, 382, 543

Lejeune, T., \& Schaerer, D. 2001, A\&A, 366, 538

Mills, B. Y., Turtle, A. J., Little, A. G., \& Durdin, J. M. 1984, Aust. J. Phys., 37, 321

Pacholczyk, A. G. 1970, Radio astrophysics. Nonthermal processes in galactic and extragalactic sources, ed. A. G. Pacholczyk (San Francisco: Freeman)

Sanduleak, N. 1970, Contributions from the Cerro Tololo Inter-American Observatory, 89

Sault, R. J., Teuben, P. J., \& Wright, M. C. H. 1995, in Astronomical Data Analysis Software and Systems IV, ed. R. A. Shaw, H. E. Payne, \& J. J. E. Hayes, ASP Conf. Ser., 77, 433

Smith, R. C., Points, S., Aguilera, C., et al. 2004, BAAS, 36, 101.08

Smith, C., Points, S., \& Winkler, P. F. 2006, NOAO Newsletter, 85, 6

Turtle, A. J., \& Mills, B. Y. 1984, Proc. Astron. Soc. Australia, 5, 537

Zaritsky, D., Harris, J., Thompson, I. B., \& Grebel, E. K. 2004, AJ, 128, 1606 